\title{
Transanal total mesorectal excision (TATME) - preliminary findings
}

\author{
Jacek Piątkowski, Marek Jackowski, Jacek Szeliga, Mariusz Nowak \\ Chair and Clinic of General, Gastroenterological and Oncological Surgery, Collegium Medicum in Bydgoszcz, Nicolaus Copernicus \\ University in Torun, Poland
}

Videosurgery Miniinv 2015; 10 (3): 495-498 DOI: $10.5114 /$ wiitm.2015.54060

\begin{abstract}
Fifteen years ago, laparoscopic surgery of rectal cancer was considered as radical in terms of oncology as open surgery. A trend to reduce surgical injury may be observed in surgery in recent years. In this paper the first experience in the new minimally invasive method of anterior rectal resection is described - through the transanal way. The procedure duration was $130 \mathrm{~min}$. The use of transanal access in rectal cancer surgery is undoubtedly an alternative method to standard laparoscopic surgery. In patients with a large tumour in the lower rectum, the technique allows a longer rectal stump to be left below the resection border.
\end{abstract}

Key words: colorectal cancer, laparoscopy, anterior resection.

\section{Introduction}

Fifteen years ago, laparoscopic surgery of rectal cancer was considered as radical in terms of oncology as open surgery. Its advantages include reduced surgical injury, shorter hospitalisation and quicker full recovery. Additionally, laparoscopy is related to lower intraoperative blood loss and fewer infectious complications, with comparable oncological results.

There are ongoing studies aiming at further reduction of surgical injury, improved quality of treatment and life comfort of patients after surgery. Therefore, a technique of anterior rectal resection via transanal access has been proposed.

\section{Case report}

A 65-year-old female patient reported to the clinic due to an extensive polypoid rectal lesion. The patient complained about rectal discomfort and chronic diarrhoea accompanied by mucus and sometimes blood. In the last 6 months the patient reported a $5 \mathrm{~kg}$ weight loss. She had not suffered from serious diseases before.

Colonoscopy presentation: A vast carpet lesion spreading from the rectosigmoid junction over the whole rectal circumference and rectal bubble and reaching the pectineal line. The lesion is $15-18 \mathrm{~cm}$ long and covers the whole circumference of the rectum. Polyp specimens revealed: villous polyp with high-grade dysplasia foci.

Computed tomography revealed thickening of the anal wall with pronounced narrowing of its lumen, normal-sized locoregional lymph nodes, and no other pathologies in the abdominal organs. The patient was qualified for surgical treatment (transanal total mesorectal excision).

After preparing the patient, she was operated on under general anaesthesia in the supine position. The procedure was performed concomitantly by 2 teams via transabdominal and transanal access. 
After insufflation of the pleural cavity with carbon dioxide, 4 trocars were introduced (in the periumbilical region, on both sides of the abdomen at the umbilicus level and in the right iliac fossa).

The inferior mesenteric artery was prepared and ligated (below the root of the left colic artery). The sigmoid colon and descending colon were released in a typical way, and then a side peritoneal incision was performed along the rectum.

After introducing a port (GelPort Laparoscopic System) into the rectum, its lumen was closed with a purse-string suture. A tattoo was made to mark the site of the rectal dissection. Then the rectum was dissected in a circular manner by means of coagulation, proximally from the purse string, revealing presacral fascia (Photos 1, 2). With upward dissection the rectum was removed with the undamaged mesorectum. The peritoneal deflection was reached

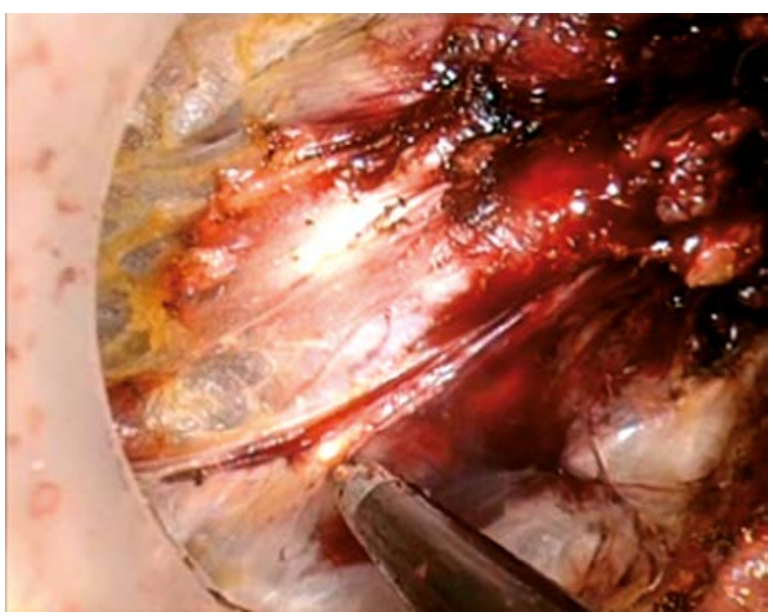

Photo 1. Dissection of mesorectum

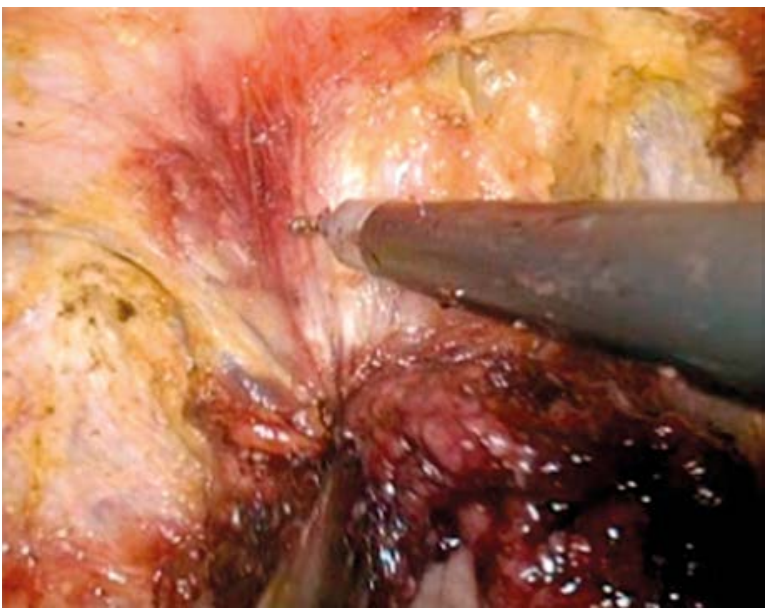

Photo 2. Dissection of frontal part of rectum basally, and after it was cut, the retroperitoneum was connected to the peritoneal cavity. The released rectum was removed through the anal pit. After the rectal excision, mucus was also removed from the rectal stump (Photo 3 ). Coloanal anastomosis was performed with single sutures.

The procedure duration was $130 \mathrm{~min}$. The patient was discharged home in a good condition on day 5 following the surgery. No complications were observed in the post-operative period.

The patient remains under ambulatory care. She has not reported any complaints so far.

\section{Discussion}

A trend to reduce surgical injury may be observed in surgery in recent years. The next step after classic laparoscopic surgery seems to be single incision laparoscopic surgery (SILS) followed by natural orifice translumenal endoscopic surgery (NOTES). However, due to technical problems, especially the latter remains in the phase of experiments and clinical trials [1].

The standard method of rectal cancer treatment is currently total mesorectal excision (TME), preferably via laparoscopic access [2].

The rectum with the mesentery is resected from the upper part inferiorly toward the bottom of the pelvis. In case of technical problems, such as a large tumour, a lesion located in the lower rectum and a narrow pelvis, rectal preparation may be very difficult and may carry the risk of intra- and post-operative complications. Another problem might be to obtain a sufficient oncological margin below the

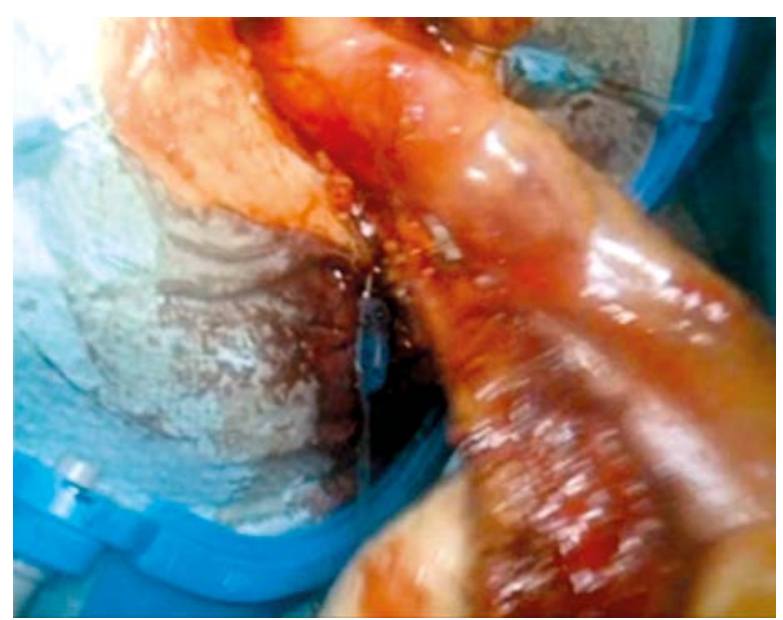

Photo 3. Rectum specimen with the tumour 
tumour. Reaching the area below the lesion with a laparoscopic stapler and performing rectal occlusion may, especially in the above cases, cause a lot of problems [3].

All this results in a relatively high percentage of conversions, which has a direct effect on complications in patients treated for rectal cancer.

The above-mentioned problems motivate surgeons to look for a new surgical technique. A breakthrough came in 2013, when Atallach et al. [4] and Lacy et al. [5] published preliminary results of a new surgical access. It was called transanal total mesorectal excision (TATME).

Also Zhang et al. [6] and Leroy et al. [7] presented a technique based on similar assumptions, which was called by Leroy "no scar transanal TME".

With a standard laparoscopic technique, the rectum is always mobilised from the abdominal side. In theory, basal access through the anal canal is nothing new. Ramos [8] and Watanabe et al. [9] tried to operate on lower rectum cancer via the anal access, but what they did was basically releasing the lower rectum via the intersphincteric access and performing manual coloanal anastomosis.

The next step was a study published by Sylla et al. [10] describing transanal rectal resection using transanal endoscopic microsurgery (TEM). The surgery was performed using a surgical proctoscope and the technique was called transanal minimally-invasive surgery (TAMIS) TME. Its principal drawback was the proctoscope structure and size, which in many cases hindered preparation and release of the rectum.

Such attempts were also made in Poland. Wałęga published a study in which he described local resection of rectal cancer together with the mesorectum. However, this was only applied to T1 tumours.

Only the introduction of new surgical methods by Lacy (the use of Gelport, concomitant operation of two teams) resulted in much wider acceptance of the new method called TATME (transanal TME).

Rectal occlusion with a suture placed below the tumour means that under visual control we obtain a completely acceptable oncological margin. Additionally, a longer rectal stump is left free of pathological lesions and the rectum does not have to be closed with several loads of the endoscopic stapler.

This often happens in lower rectum cancers and results in a high risk of anastomotic leak. It also goes without saying that the quality of life of patients with the anastomosis $6 \mathrm{~cm}$ away from the rec- tal margin is much better than that of those whose anastomosis is located lower [2].

After circular dissection of the anal wall, gas insufflation causes easier removal of the mesorectum without the risk of damaging it. Perfect visibility of the perianal area reduces the risk of damaging autonomic nerves and facilitates manoeuvring in the so-called "holy plane". It is even more clear if we use visual tracking in a 3D technique. In most cases, transanal access also allows direct assessment of the quality of anastomotic connection performed with a circular stapler.

What is also important is the fact that concomitant operation by two teams (one via transabdominal access and the other via transanal access) is much shorter, which is likely to reduce the number of post-operative complications and early rehospitalisations [5].

All the above-mentioned advantages of the transanal method mean that it will probably become a standard procedure for treating cancer of the mid and lower rectum in the future. Results obtained so far are really promising $[2,5,10]$.

However, the groups of patients are still very small. In our clinic, only 3 such procedures have been conducted so far in the period of March-May 2015, and the post-operative observation period was not long enough to make far-reaching conclusions.

\section{Conclusions}

The use of transanal access in rectal cancer surgery is an alternative method to standard laparoscopic surgery. In patients with a large tumour in the lower rectum, the technique allows a longer rectal stump to be left below the resection border. Due to small groups of patients and a short observation period, the method requires further studies.

\section{Conflict of interest}

The authors declare no conflict of interest.

\section{References}

1. Velthuis $S$, van den Boezem PB, van der Peet DL, et al. Feasibility study of transanal total mesorectal excision. Br J Surg 2013; 100: 828-31.

2. Heald RJ. A new solution to some old problems: transanal TME. Tech Coloproctol 2013; 17: 257-8.

3. Ferko A, Orhalmi J, Dusek T, Chobola M. Higher risk of incomplete mesorectal excision and positive circumferential margin 
in low rectal cancer regardless of surgical technique. Videosurgery Miniinv 2014; 9: 569-77.

4. Atallah S, Albert M, deBeche-Adams T, et al. Transanal minimally invasive surgery for total mesorectal excision (TAMIS-TME): a stepwise description of the surgical technique with video demonstration. Tech Coloproctol 2013; 17: 321-5.

5. Lacy AM, Adelsdorfer C, Delgado S, et al. Minilaparoscopy-assisted transrectal low anterior resection (LAR): a preliminary study. Surg Endosc 2013; 27: 339-46.

6. Zhang H, Zhang YS, Jin XW, et al. Transanal single-port laparoscopic total mesorectal excision in the treatment of rectal cancer. Tech Coloproctol 2013; 17: 117-23.

7. Leroy J, Barry BD, Melani A, et al. No-scar transanal total mesorectal excision: the last step to pure NOTES for colorectal surgery. Arch Surg 2012; 19: 1-5.

8. Ramos JR. Laparoscopic very low anterior resection and coloanal anastomosis using the pull-through technique. Dis CoIon Rectum 1995; 38: 1217-9.

9. Watanabe M, Teramoto T, Hasegawa H, Kitajima M. Laparoscopic ultralow anterior resection combined with per anum intersphincteric rectal dissection for lower rectal cancer. Dis Colon Rectum 2000; 43 (Suppl): S94-7.

10. Sylla P, Rattner DW, Delgado S, Lacy AM. NOTES transanal rectal cancer resection using transanal endoscopic microsurgery and laparoscopic assistance. Surg Endosc 2010; 24: 1205-10.

Received: 14.07.2015, accepted: 21.07.2015. 